\title{
Age-Related Hemispheric Asymmetry in Object Discrimination*
}

\author{
Peter Gerhardstein ${ }^{1}$, Mary A. Peterson ${ }^{1}$, and Steven Z. Rapcsak ${ }^{2}$ \\ ${ }^{1}$ Department of Psychology, University of Arizona, Tucson, AZ, ${ }^{2}$ Department of Neurology, University of \\ Arizona, and Neurology Service, Tucson Veterans Administration Medical Center
}

\begin{abstract}
Young and elderly observers judged whether two sequentially presented images in either the left or right visual field (LVF or RVF) were the same or different. The two objects depicted were always from the same entry-level category, but could differ only in viewpoint (a "same" trial--observers were instructed to ignore viewpoint changes) and/or in exemplar (a "different" trial). Young observers showed no difference in sensitivity across visual fields. Elderly observers were less sensitive overall than young observers, and were less sensitive to stimuli presented in the LVF-RH than in the RVF-LH. The results of this experiment support the hypothesis that one type of visual cognitive functioning declines with age faster in the right hemisphere than in the left hemisphere.
\end{abstract}

The brain is divided into two hemispheres that, in terms of their gross anatomy, are approximate mirror images of one another. Despite this general similarity in the structure of the two hemispheres, there are many dissimilarities in function. For instance, it has long been acknowledged that the left hemisphere is primarily responsible for processing language, whereas the right hemisphere is primarily responsible for processing spatial information (e.g., De Renzi, 1982; Milner, 1975; Nebes, 1974). Beyond these general notions about the differential lateralization of verbal and spatial processing, research has revealed clear evidence that specific visual and spatial skills are differentially lateralized. For example, neuropsychological and behavioral studies have shown that the left hemisphere specializes in processing local aspects of a visual stimulus, and the right hemisphere specializes in processing global aspects (Lamb \& Robertson, 1988; Robertson, Lamb, \& Zaidel, 1993). In addition, Kosslyn and others have found evidence that the left hemisphere processes categorical information best, whereas the right hemisphere processes more precise coordinate information best (Kosslyn, 1987; Kosslyn, Maljkovic, Hamilton, Horwitz, \& Thompson, 1995; Marsolek, 1995). Furthermore, Sergent, Ohta, and MacDonald (1992) reported a left-hemisphere advantage for categorizing objects as living versus nonliving, and a right-hemisphere advantage for identifying faces. None of the processing asymmetries mentioned above is absolute, however. In every case, both hemispheres are capable of performing both types of function, but one does so relatively faster and/or relatively more accurately than the other.

\section{Aging and the Two Hemispheres}

In addition to such specialization of function as described above, there may be other differences between the two hemispheres; and in particular, the two hemispheres may be differentially susceptible to the aging process. For example, it has been proposed that the right hemisphere declines earlier with age than the left hemisphere (Albert

\footnotetext{
* Address correspondence to: Peter Gerhardstein, Department of Psychology, Binghamton University, PO Box 6000, Binghamton, NY 13902, USA. E-mail: gerhard@binghamton.edu. Accepted for publication: December 8, 1997.
} 
\& Moss, 1988; Goldstein \& Shelley, 1981). This hypothesis was advanced because performance on the verbal component of the Wechsler Adult Intelligence Scale (WAIS; Wechsler, 1958), declines less with age than does performance on the spatial component of the same test (Goldstein \& Shelley, 1981; Levy, 1969).

There are problems with the WAIS task, however. For example, verbal skills are measured via tests of an overlearned vocabulary, whereas spatial skills are measured via novel pattern-matching tasks. Performance on tasks assessing overlearned material may deteriorate later with aging regardless of whether they are verbal or spatial. Accordingly, Meudell and Greenhalgh (1987) used a task developed by Heim (1970) that requires the use of verbal and spatial reasoning rather than recall of previously learned information. Using these reasoning tasks, Meudell and Greenhalgh found, consistent with the differential aging hypothesis, that elderly individuals, relative to younger participants, exhibited larger deficits on spatial tasks than on verbal tasks. In addition, clinical testing of normal elderly individuals has produced a body of evidence that visual-spatial skills decline faster than language skills with age (e.g., Klisz, 1978)

Recently, Lawrence, Myerson, and Hale (1997) reported additional evidence in support of the differential aging hypothesis, assessing the relative processing time required for verbal $(\mathrm{LH})$ and spatial tasks $(\mathrm{RH})$. They found that verbal processing time increased by about $50 \%$ over the age range of 20-90 years, whereas processing time on several spatial tasks increased exponentially, increasing by $500 \%$ over the same age range. These results are supported by previous reports of a general age-related slowing of processing in both verbal and spatial tasks (e.g., Hale, Lima, \& Myerson, 1991, for verbal and spatial tasks, respectively; Myerson, Ferraro, Hale, \& Lima, 1992). Indeed, Lima, Hale, and Myerson (1991) performed a meta-analysis of previous studies and found that processing in nonlexical, spatial tasks slowed more rapidly with age than did processing in lexical, or verbal tasks. Given the evidence that the RH specializes in spatial processing and the LH specializes in verbal processing, these results are consistent with the differential aging hypothesis.

Despite the convergence of results using different means of assessing verbal and spatial skills, comparisons across verbal and spatial tasks may not be ideal for reaching conclusions about the relative efficiency of the two hemispheres. This is because it may not be possible to equate the difficulty of verbal and spatial tasks. Elias and Kinsbourne (1974) argued that spatial tasks are likely to be more complex, and hence, more difficult, than verbal tasks. Suppose that aging results in an overall (nonhemispherespecific) decline in cognitive abilities. Such a decline would be more likely observed in difficult tasks (e.g., on this view, spatial, right-hemisphere-specific tasks) than in simpler tasks (e.g., on this view, verbal, left-hemisphere-specific tasks). Given that there is no known method for equating spatial and verbal tasks for difficulty, no firm conclusions about the differential decline of the two hemispheres with age can be drawn on the basis of comparing performance on verbal and spatial tasks.

The differential aging hypothesis can be tested without settling the question of whether or not spatial and verbal tasks can be equated for difficulty, by comparing the performance of young and elderly observers on a single task performed under lateralized presentation conditions. Under lateralized presentation conditions, task-relevant stimuli are briefly presented in either the left visual field (LVF) or the right visual field (RVF). It is assumed that stimuli presented to one visual field will be processed first, and more completely, by the contralateral hemisphere; thus, LVF presentation maximizes right hemisphere (RH) processing, and RVF presentation maximizes left hemisphere (LH) processing. (For a review of the assumptions underlying the use of this technique, see Clarke \& Miklossy, 1990; Kosslyn \& Koenig, 1992)

A few investigators have tested the differential aging hypothesis using a single task; some of the results have supported the differential aging hypothesis, whereas others have failed to support it. An example of the latter type of result is that reported by Nebes, Madden, and Berg (1983), who used two types of tasks: a verbal 
task that required reading the name of a number, and a "spatial" task that required reading the time depicted on a numberless clock face. For both young and elderly observers, Nebes and colleagues found that the verbal task was performed better when initial presentation was to the RVF-LH rather than to the LVF-RH, and that the spatial task was performed better when initial presentation was to the LVF-RH rather than to the RVF-LH. Thus, for both young and elderly observers, the classic expected pattern of cerebral asymmetries was obtained. More importantly for the differential aging hypothesis, the asymmetries in performance were equivalent for both young and elderly observers, contrary to the hypothesis that the right hemisphere declines more quickly with age than the left hemisphere.

Certainly, the results reported by Nebes et al. (1983) are inconsistent with the hypothesis that all right hemisphere tasks will reveal evidence for a decline in right hemisphere processing. This conclusion should not be too surprising, however, because the original hypothesis was probably drawn too broadly. A more restricted hypothesis that supposes that certain right hemisphere structures will reveal age-related impairments before their corresponding left hemisphere structures might be more reasonable. Of course, it is not clear which brain structures are involved in the verbal and spatial versions of the clock-time task, but the Nebes et al. results suggest that the aging process may not differentially affect the left and right brain regions mediating performance on these tasks. Conclusions regarding other tasks, and other brain areas, require further research.

More recently, other researchers (Polster \& Rapcsak, 1994; Stark \& Coslett, 1993) have reported evidence consistent with the proposal that at least some structures in the right hemisphere do age faster than the corresponding structures in the left hemisphere. These investigators used brief presentations of hierarchical stimuli, such as those shown in Figure 1, in which a large letter is formed using many small letters. On different blocks of trials, observers were instructed to search for targets at either the large, global level or at the small, local level. Previous re- search using these tasks has revealed a global advantage overall, suggesting that the global level is processed faster than the local level (Boles, 1984; Lamb \& Robertson, 1988; Navon, 1977; Robertson et al., 1993). In addition, the right hemisphere has been shown to be specialized for global processing, and the left hemisphere for local processing (e.g., Lamb \& Robertson, 1988). More specifically, Lamb and Robertson have shown that damage to posterior right hemisphere structures can result in a local, rather than a global, advantage in the perception of hierarchical stimuli. Polster and Rapcsak (1994) compared the performance of elderly and young observers viewing hierarchical stimuli. They found the expected global processing advantage for young participants, but they found a local processing advantage for elderly participants. Thus, Polster and Rapcsak found that a shift occurred as a function of age from an advantage attributable to $\mathrm{RH}$ processing (global processing) to an advantage attributable to $\mathrm{LH}$ processing (local processing). These data, and similar data reported by Stark and Coslett (1993), are consistent with the hypothesis that certain structures in the right hemisphere may be damaged more by the normal aging process than the corresponding structures in the left hemisphere. Lamb and Robertson's work suggests that posterior right hemisphere might be the locus of this damage.

The current study was designed to investigate whether a task employing pictures of objects rather than letters might reveal an age-related change in the processing capabilities of the left and right hemispheres. We did not assess local versus global processing; instead, we assessed the observer's ability to judge whether two successively presented pictures depicted the same or a different exemplar of a basic-level category. This task was used previously by Biederman, Gerhardstein, Cooper, and Nelson (1997). Young observers viewed, in either the LVF or the RVF, a pair of drawings of objects presented sequentially with a masked interstimulus interval and judged whether the two depicted objects were the same or different. The paired objects were either depicted from the same three-dimensional vantage point, or from vantage points that 


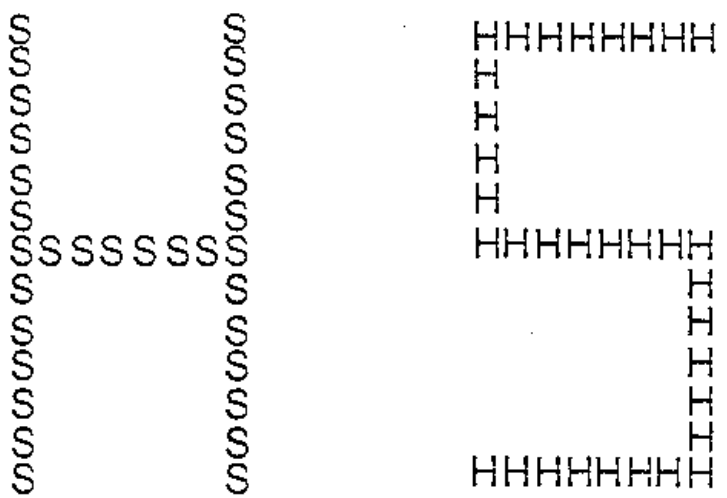

Fig. 1. An example of the hierarchical stimuli used to test global versus local processing. Large letters (global stimuli) are constructed from small letters (local stimuli). Observers are instructed to look for a target letter (e.g., H or S) at either the global or the local level. At the other level, stimuli are either consistent, inconsistent, or neutral. The stimuli shown here are inconsistent stimuli if the targets are $\mathrm{H}$ and $\mathrm{S}$.

differed by $30^{\circ}$ or $60^{\circ}$. The depicted objects in each pair were from the same entry-level category and had the same basic level name (e.g., both were ladders), but they were not always the same exemplar (e.g., one might be a step-ladder and the other, an extension ladder; see Fig. 2). Observers were to respond "same" when the two successive drawings depicted the same exemplar, regardless of the viewpoint from which it was depicted; and they were to respond "different" when the two successive drawings depicted a different exemplar, again, regardless of viewpoint.

Using this task, Biederman et al. (1997) found no indication of either right- or left-hemisphere superiority when they tested young participants. The failure to find hemispheric differences in young participants' performance is in general agreement with other previous studies using similar object-recognition tasks (Biederman \& Cooper, 1991b; Levine \& Banich, 1982). In the present study, we sought to replicate the Biederman et al. results with a group of young participants, and to examine whether or not hemispheric differences emerged in a group of elderly participants. If the differential aging hypothesis is correct, evidence for RH impairments in the elderly participants might emerge even in tasks that show no hemispheric superior- ity in the young, and would take the form of poorer performance in the LVF-RH than in the RVF-LH.

\section{METHOD}

\section{Subjects}

The participants were 32 individuals, 16 ( 8 male, 8 female) of whom were young, and 16 (3 male, 13 female) of whom were elderly. The mean age of the young participants was 19.9 years (range: 17-26 years), and the mean age of the elderly participants was 69.7 years (range: $63-78$ years). The young participants were students at the University of Arizona who received either class credit or $\$ 5.00$ in return for their participation. The elderly participants were members of a retirement community in southern Arizona who were recruited through a volunteer program at the University of Arizona Medical Center. They were reimbursed for their parking costs while serving as participants (average $=\$ 5.00)$.

All participants were right-handed; the young participants scored 50 or more on the Edinburgh Handedness Inventory (Oldfield, 1971); the elderly participants scored 50 or more on a questionnaire containing 7 of the 12 items on the Edinburgh and an additional 7 items. The questionnaire was scored by calculating a ratio of $\left[(R-L) /(R+L)^{*} 100\right]$, where $R=$ the number of tasks for which a participant indicated he/she was right-handed, and $L=$ 


\section{Types of "Same" trials}

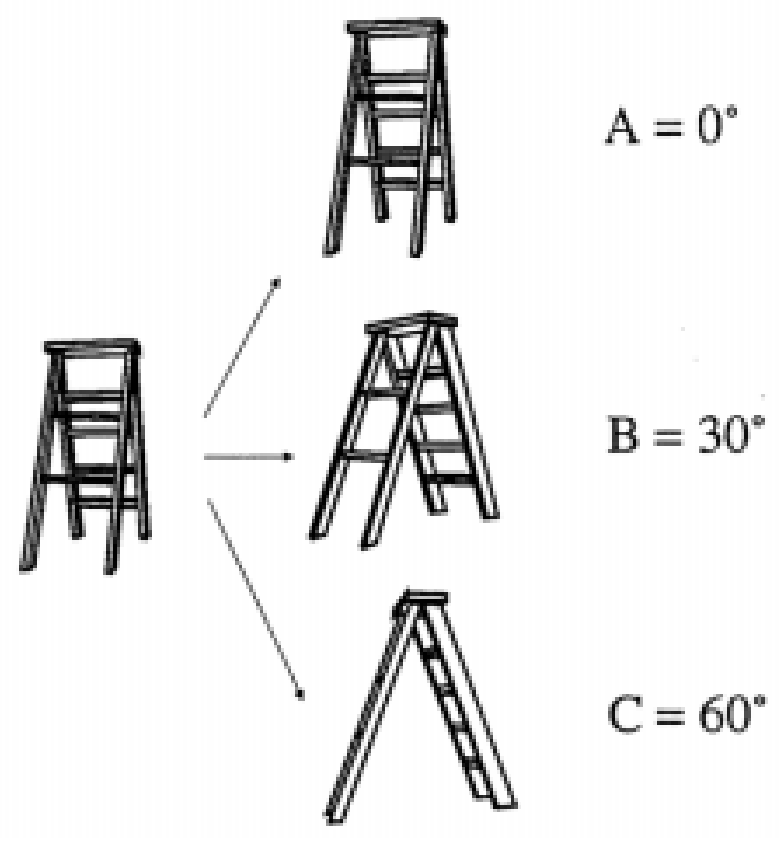

\section{Types of "Different" trials}

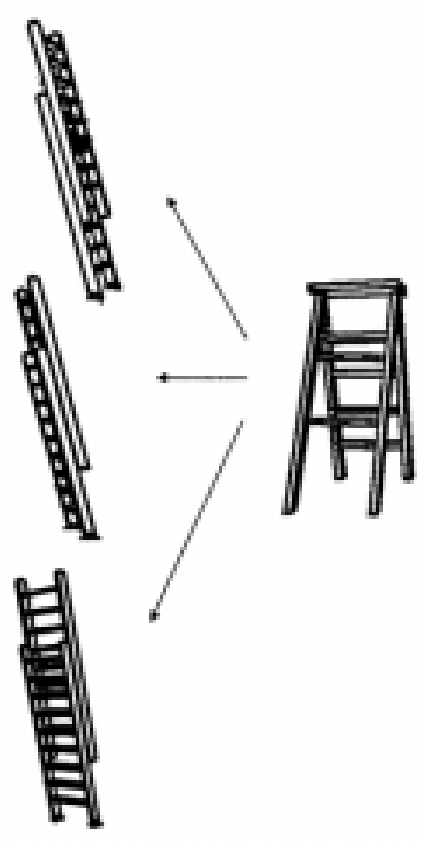

Fig. 2. Illustration of the possible object pairings for one pair of stimuli. Note that the first stimulus shown could be either A or C.

the same for left-handed. Thus, calculated handedness scores could run from -100 (extremely lefthanded) to +100 (extremely right-handed). A score of 50 means that the participant indicated that the right hand was the dominant hand for at least $75 \%$ of all tasks in the questionnaire. Persons with lefthanded parents or siblings were excluded.

The young participants all had normal or corrected-to-normal vision; the elderly participants all met the criterion that they could "read a newspaper without difficulty", with or without glasses (if glasses were required, they were worn during the test session). Additionally, all of the elderly participants achieved a score of $86 \%$ or greater (mean = $94.2 \%$ ) on a dot-localization task prior to testing.

\section{Stimuli and Apparatus}

Line drawings of 24 pairs of common objects were used as stimuli. The pairs consisted of two objects with the same basic-level category name, but with a different shape; the two objects were therefore different exemplars of a basic-level category (see Fig. 2; Appendix A). For example, two ladders are shown in Figure 2; one is a step ladder, and the other is an extension ladder. Three views (A, B, and $\mathrm{C}$ ) were produced for each object, with a $30^{\circ}$ rotation in depth between views, as can be seen in Figure 2. View A was generally a front-right view of the object, altered as needed to allow orientation changes that would not result in highly self-occluded views at the other orientations used (i.e., no highly unusual views were used). Stimuli were sized to fit within a circle $12 \mathrm{~cm}$ in diameter and were presented at $150-\mathrm{cm}$ viewing distance, for a maximum effective visual angle of $4.5^{\circ}$. The stimuli were presented in one of the four quadrants of the screen, two to the left, and two the right of fixation. The center of each object was located $4.0^{\circ}$ from fixation $\left(3.22^{\circ}\right.$ horizontal, $2.48^{\circ}$ vertical). The closest feature of any object was at least $1.76^{\circ}$ from the center of the screen. The lines of the stimuli were black, drawn on a white screen. The pictures of the objects were presented on a Sampo 19inch monochrome monitor $(1024 \times 768$ pixels, refresh rate $75 \mathrm{~Hz}$ ), controlled by a Macintosh IIx computer. 


\section{Procedure}

Participants were instructed to press a mouse to begin each trial. When the mouse was pressed, a black central fixation dot (spanning $0.75^{\circ}$ ) was presented for $500 \mathrm{~ms}$ on a white screen. Participants were instructed to look at the dot, and to keep their eyes focused on that location after the dot disappeared. After the dot disappeared, a picture of an object was presented for $250 \mathrm{~ms}$ in one of the four corners of the screen, and was followed by a dense mask of random lines presented for 133 $\mathrm{ms}$. A second object was then presented for $250 \mathrm{~ms}$ in the same location as the first (see Fig. 3 for an illustration of the sequence). The second object was either the same as the first (viewed from one of three vantage points) or different from the first in that it was a different exemplar of the same category. On trials on which the two exposures were of the same object, the first stimulus (prior to the mask) was presented in view $A$ or view $C$, and the second exposure could be any of the three views of the object (see Fig. 2), resulting in orientation changes of $0^{\circ}, 30^{\circ}$, and $60^{\circ}$ between the first and second exposures on "same" trials. The same three amounts of orientation change occurred between exposures on "different" trials, with the "orientation change" in those cases being arbitrarily defined as the amount by which the orientation would have differed, had the objects been the same.

\section{Task}

Participants were instructed to decide whether the two objects shown successively on a single trial were the same or different, and to indicate their choice by pressing one of two buttons on a button box, using their right hand. Participants were instructed that when the two objects were the same, the viewpoint might change between the first and second presentation of the object. They were told to ignore such changes and respond "same" in that situation. They were told to call the objects "different" only if the two were physically different objects or would not look the same from any viewpoint. They were also told that the two objects would have the same basic name on all trials. Par-

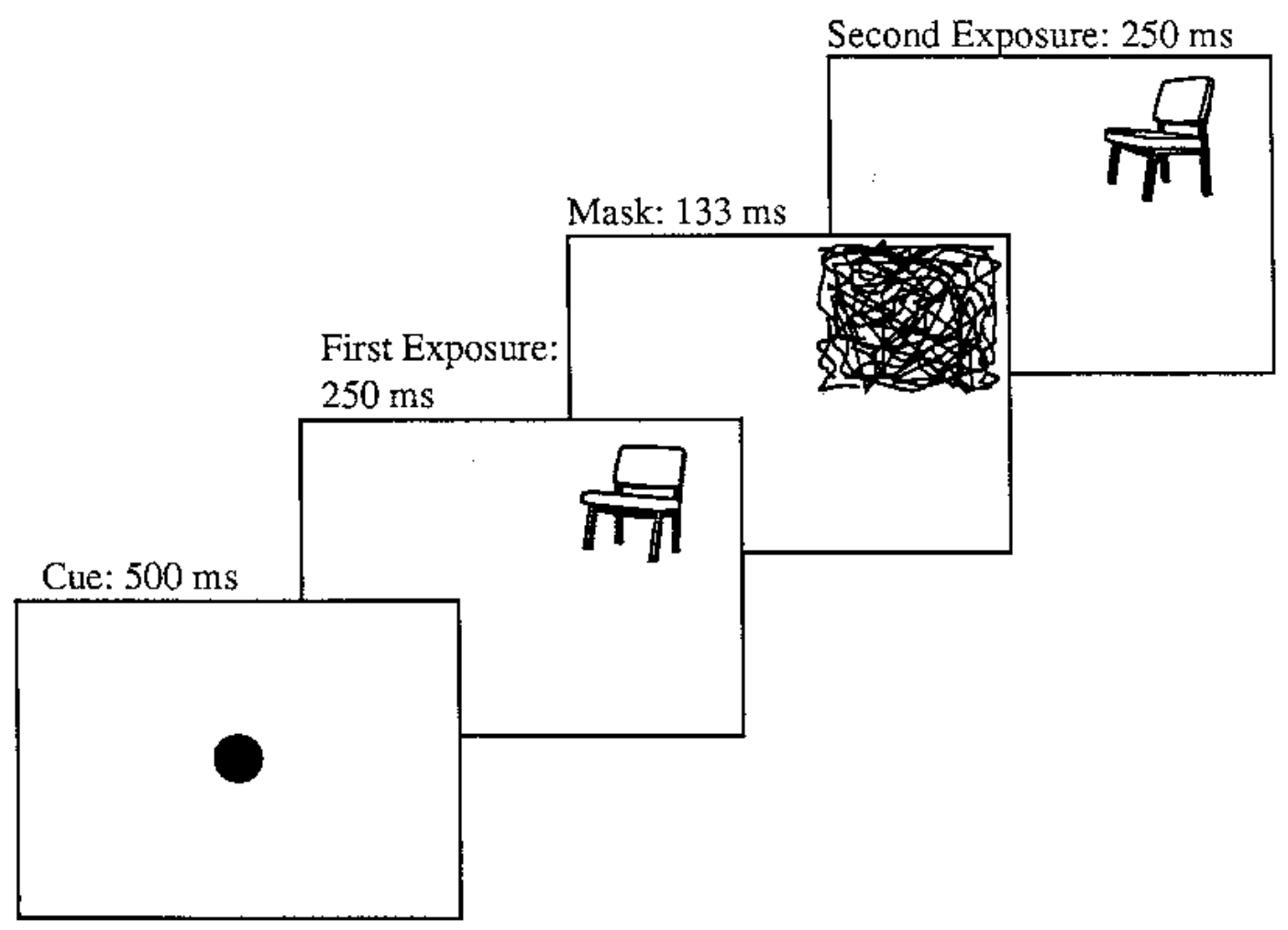

Fig. 3. Illustration of a single trial; a $30^{\circ}$ change in orientation occurs between the first and second exposures of the stimulus (the correct response for this trial is "same"). 
ticipants were given 15 trials of practice, with feedback, using objects that did not appear as stimuli in the experiment. No feedback was given during the experiment itself.

Participants were given $5 \mathrm{~s}$ in which to respond. Trials on which no response was received within 5 s were recorded as "time-out" errors. The button box was connected to a National Instruments timing board (S/be NB-TIO-10), which provided millisecond timing accuracy.

\section{Design}

The design covered Quadrant (4) $\times$ Orientations $\left(0^{\circ}, 30^{\circ}, 60^{\circ}\right) \times$ Condition (same or different), resulting in 24 condition "cells". Participants saw six objects in each of the 24 cells, for a total of 144 trials, meaning that each object was seen six times by each participant (three "same" and three "different" trials). The six cells in which a given object was viewed were counterbalanced across participants, so over 4 participants, each object was presented in each cell. The presentation order was then reversed and the above conditions repeated for the next four participants. This sequence was repeated for an additional eight participants.

\section{RESULTS}

For each visual field, we collapsed across the trials on which stimuli appeared in the upper and lower quadrants. The mean proportions of accurate "same" and "different" responses obtained for each group (elderly vs. young) for each condition of orientation change and hemisphere were calculated. One elderly participant responded "same" on every trial. It is unclear whether this participant misunderstood the instructions or simply was unable to perform the task, but this participant's data were not in- cluded in any of the analyses presented below. Hence, the final data set includes an unequal number of young and elderly participants.

The accuracy measures are shown in Table 1. For the young and the elderly participants, accuracy was higher on same trials than on different trials and accuracy declined as the orientation change increased from $0^{\circ}$ to $60^{\circ}$. For the young, the greatest decline in accuracy on same trials occurred between the $0^{\circ}$ and $30^{\circ}$ conditions. For the elderly participants, the greatest decline in accuracy on same trials occurred between the $30^{\circ}$ and $60^{\circ}$ conditions. For the $0^{\circ}$ condition, the accuracy difference between same trials and different trials was larger for the elderly participants than for the young participants.

Table 1 shows that both young and elderly participants were less likely to be accurate on different trials than on same trials. In other words, observers incorrectly responded "Same" on a substantial proportion of different trials. These incorrect responses are called "false alarms" (equal to ( 1 - proportion correct on different trials)). By jointly considering both false alarms and hits (proportion correct on same trials), a measure of an observer's true sensitivity can be derived, even if the observer is biased to respond "same" in the face of uncertainty, as might be the case when lower accuracy is obtained on different trials. Accordingly, we used a sensitivity measure to summarize performance. Sensitivity $\left(d^{\prime}\right)$ was defined as

$$
d^{\prime}=\text { Hit rate }- \text { False Alarm rate }
$$

where Hit rate $=$ proportion of trials on which an observer correctly responded "Same" on same

Table 1. Accuracy by Age for Each Orientation Change.

\begin{tabular}{|c|c|c|c|c|c|c|c|c|c|c|c|c|}
\hline \multirow[b]{3}{*}{ Resp. } & \multicolumn{6}{|c|}{ Young } & \multicolumn{6}{|c|}{ Elderly } \\
\hline & \multicolumn{3}{|c|}{ LH } & \multicolumn{3}{|c|}{ RH } & \multicolumn{3}{|c|}{ LH } & \multicolumn{3}{|c|}{$\mathrm{RH}$} \\
\hline & $0^{\circ}$ & $30^{\circ}$ & $60^{\circ}$ & $0^{\circ}$ & $30^{\circ}$ & $60^{\circ}$ & $0^{\circ}$ & $30^{\circ}$ & $60^{\circ}$ & $0^{\circ}$ & $30^{\circ}$ & $60^{\circ}$ \\
\hline Same & .95 & .88 & .86 & .96 & .87 & .85 & .94 & .90 & .73 & .91 & .82 & .78 \\
\hline Diff. & .88 & .81 & .69 & .86 & .77 & .69 & .78 & .77 & .66 & .69 & .66 & .60 \\
\hline
\end{tabular}

Note LH = Left Hemisphere; RH = Right Hemisphere, Resp. $=$ Response; Diff. $=$ Different 
trials, and False Alarm rate $=$ proportion of trials on which an observer incorrectly responded "Same" on different trials. Sensitivity measures have a long history in perception research (for review, see Engen, 1971), and have been successfully extended into more cognitive research areas (see Snodgrass \& Corwin, 1988).

The actual range of the sensitivity measure $d^{\prime}$ is -1.0 to +1.0 . Functionally speaking, however, it ranges from 0.0 (no sensitivity, where the Hit rate equals the False Alarm rate) to 1.0 (perfect sensitivity, where the Hit rate $=1.0$ and the False Alarm Rate $=0.0$ ). Note that a sensitivity measure of 0.0 is the equivalent of a $50 \%$ correct response rate, or guessing.

Trials on which the participant failed to give a response were not included in calculating the sensitivity, because these "time-out errors" could not be coded as either "same" or "different" responses. The young participants made 16 such time-out errors over all trials and all participants (less than $1 \%$ of all trials); 8 time-out errors were made in each condition of visual field presentation and hemisphere. The elderly participants made 57 time-out errors across all trials and all participants (2.5\% of all trials); of these, 32 occurred in the LVF-RH condition and 25 occurred in the RVF-LH condition. The effect of age was significant in a planned comparison: Elderly participants made significantly more time-out errors than did the young participants. An interaction between hemisphere and orientation change was significant as well; $F(2,58)=9.6, p<.0002$ : A larger increase in errors among the elderly observers relative to the young observers occurred in the LVF-RH condition than in the RVF-LH condition.

\section{Sensitivity}

An ANOVA with one between-subjects factor (age: elderly vs. young), and two within-subjects factors (hemisphere of presentation: LVF-RH vs. RVF-LH; and orientation change: $0^{\circ}, 30^{\circ}$, $60^{\circ}$ ) was performed on the sensitivity data. A main effect of age was obtained, $F(1,29)=$ $14.35, p<.001$, indicating that the young participants were more sensitive overall than the elderly participants. This finding reinforced the difference in time-out errors committed by the young versus the elderly participants. Main effects of orientation change, $F(2,58)=51.26, p<$ .0001 , and hemisphere, $F(1,29)=6.11, p<.02$, were obtained as well. None of the interaction effects was significant in this ANOVA, however. Nevertheless, planned comparisons were conducted within each of the two groups to test the differential aging hypothesis. These planned comparisons indicated that for the young participants, sensitivity did not differ across the two hemispheres, $F<1$, replicating Biederman et al. (1997). For the elderly participants, however, sensitivity in the left hemisphere was significantly greater than in the right hemisphere, $F(1$, 14) $=6.49, p<.03$. This greater sensitivity in the $\mathrm{LH}$ relative to the $\mathrm{RH}$ was obtained for the elderly participants in the $0^{\circ}$ and $30^{\circ}$ conditions ( $\mathrm{p}<.02$ and .01 , respectively), but not in the $60^{\circ}$ condition, where LH and RH sensitivity were approximately equally low (see Fig. 4).

This interpretation was supported by a second ANOVA on age, hemisphere, and orientation in which only the $0^{\circ}$ - and $30^{\circ}$-data were included. A significant age by hemisphere interaction emerged; $F(1,29)=6.17, p<.02$. For elderly participants, sensitivity was lower in the RH than in the LH when the task required discriminating between two stimuli that differed from one another by $30^{\circ}$ or less.

\section{DISCUSSION}

The present study compared performance in young and elderly participants on a single task performed under lateralized presentation conditions. The results obtained with the young participants replicated those previously reported by Biederman et al. (1997), in that no hemispheric superiority was obtained. There might be some concern about the failure to find hemispheric differences in the present experiment because we neither measured eye movements nor gave the observers a task to keep their eyes at fixation between the first and second object presented on each trial. We did not use a fixation task in the present experiment because the elderly participants were tested as controls for brain-damaged 


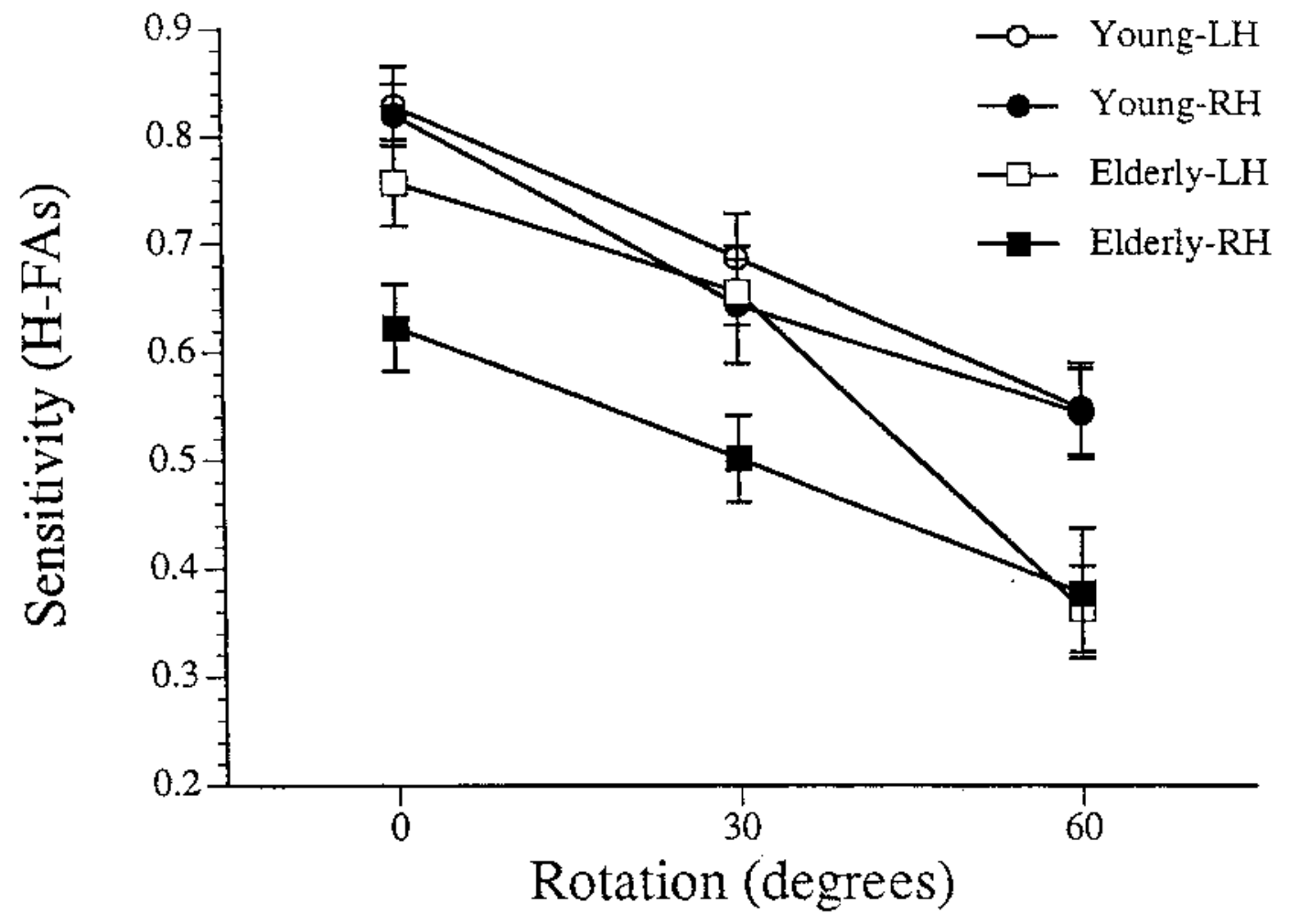

Fig. 4. Sensitivity of the two groups, young and elderly, in the LVF-RH and RVF-LH presentation conditions, at all three orientations used in the experiment. The sensitivity measure accounts for performance on both same and different trials. Error bars indicate $+/-1$ SE.

patients, who were unable to perform the fixation task.

We do not think eye movements were responsible for the failure to find hemispheric differences in young observers in the present experiment, however, because Biederman and Cooper (1991a) found the same results (absence of hemispheric differences) both when observers engaged in a secondary task requiring fixation, and when they had no task at fixation.

A difference between the two hemispheres emerged in the performance of the elderly participants, however: For the $0^{\circ}$ and $30^{\circ}$ conditions, elderly participants' sensitivity in the LVF-RH was considerably lower than in the RVF-LH. Under RVF-LH conditions, elderly participants' sensitivity in the $0^{\circ}$ and $30^{\circ}$ condi- tions did not differ from that of the young participants. A similar pattern was found in the analysis of time-out errors (trials on which a participant did not give an answer). Thus, the present results, indicating decreased sensitivity in the RH of elderly participants as compared to the $\mathrm{LH}$, are consistent with previous evidence of an age-related decline in $\mathrm{RH}$ processing (e.g., Hale et al., 1991; Myerson et al., 1992; Polster \& Rapcsak, 1994; Stark \& Coslett, 1993).

The object discrimination task that we employed requires the comparison of objects across different rotations around a vertical axis. Neuropsychological studies have indicated that difficulties in recognizing objects across different views typically emerge as a consequence of posterior RH lesions (e.g., Humphreys \& Riddoch, 
1984; Riddoch \& Humphreys, 1986; Warrington, 1982; Warrington \& James, 1988; Warrington \& Taylor, 1978). On the hypothesis that the $\mathrm{RH}$ declines faster than the $\mathrm{LH}$ as a function of aging, the present results can be taken as consistent with the results of those previous studies of patients with focal brain damage. In the previous studies, RH damage occurred as a function of focal brain pathology, whereas in the present study, it is the result of a normal aging process.

Of course, the exact nature of the object recognition processes involved in the object discrimination task are unknown. It is noteworthy that in the present experiment, evidence for the differential aging hypothesis was obtained only for the $0^{\circ}$ - and $30^{\circ}$-orientation-change conditions, and not for the $60^{\circ}$-orientation-change conditions. In the $60^{\circ}$-orientation-change conditions, the performance of elderly participants was approximately equivalent in the right and left hemispheres; and for both of these conditions performance was impaired relative to the performance of young participants. Thus, in addition to the right-hemisphere-specific decline in performance obtained in the $0^{\circ}$ and $30^{\circ}$ conditions, a non-hemisphere-specific decline in performance was observed in the $60^{\circ}$ condition for elderly observers relative to young observers.

The cause of the difference in performance in the $60^{\circ}$ condition must be determined. The task is certainly more difficult in the $60^{\circ}$ condition than in the other conditions, as evidenced by the fact that, for both the elderly and the young groups, and in both hemispheres, sensitivity declined as orientation change increased. As discussed earlier, effects of aging are more likely to be evident on more difficult tasks, or on more difficult conditions within a single task. Alternatively, the poor performance of the elderly participants relative to the young participants in the $60^{\circ}$ conditions may indicate that the object discrimination task employed in this experiment draws upon different resources in the $60^{\circ}$-orientation-change condition than in the $0^{\circ}$ - and $30^{\circ}$ orientation-change condition. It is noteworthy that the accuracy of the elderly participants' "same" responses declined dramatically between $30^{\circ}$ and $60^{\circ}$ in the RVF-LH condition, but not in the LVF-RH condition. We are currently investigating the degree to which different component skills are involved in performing this task in the different orientation conditions, and the degree to which these different skills are affected by aging.

The source of elderly participants for our experiment was extremely limited, and thus we were forced to accept an unequal number of male and females in our elderly group; this problem precluded an analysis of gender effects in the present experiment. While no gender-related hemispheric differences were observed in a figure/ground discrimination task with $6 \mathrm{fe}$ male and 6 male (including the 3 from the present study) elderly participants, future tests should manipulate gender directly, as differential aging across gender in this task is certainly a possibility.

In summary, the present study yielded support for the differential aging hypothesis by comparing the performance of young and elderly observers on a discrimination task involving two successively presented objects drawn from the same basic-level category. This task did not appear to be lateralized among the young participants. Nevertheless, it appears to be sensitive to right hemisphere decline in the elderly participants, whose performance in the $0^{\circ}$ and $30^{\circ}$ conditions was impaired relative to that of young participants only under LVF-RH presentation conditions.

\section{REFERENCES}

Albert, M.S., \& Moss, M.B. (1988). Geriatric neuropsychology. New York: Guilford Press.

Biederman, I., \& Cooper, E.E. (1991a). Evidence for complete translational and reflectional invariance in visual object priming. Perception, 20, 585-593.

Biederman, I., \& Cooper, E.E. (1991b). Object recognition and laterality: Null effects. Neuropsychologia, 7, 685-694.

Biederman, I., Gerhardstein, P., Cooper, E.E., \& Nelson, C.A. (1997). High level object recognition without an anterior inferior temporal lobe. Neuropsychologia, 35, 271-287.

Boles, D.B. (1984). Global versus local processing: Is there a hemispheric dichotomy? Neuropsychologia, 22, 445-455.

Clarke, S., \& Miklossy, J. (1990). Occipital cortex in man: Organization of callosal connections, related 
myleo- and cytoarchitecture, and putative boundaries of functional visual areas. Journal of Comparative Neurology, 298, 188-214.

De Renzi, E. (1982). Disorders of space exploration and cognition. New York: John Wiley \& Sons.

Elias, M.F., \& Kinsbourne, M. (1974). Age and sex differences in the processing of verbal and nonverbal stimuli. Journal of Gerontology, 29, 162-171.

Engen, T. (1972). Psychophysics I. Discrimination and detection. In J.W. Kling \& L.A. Riggs (Eds.), Woodworth \& Schlosberg's experimental psychology (3rd ed., pp. 11-46). New York: Holt, Rinehart $\&$ Winston.

Goldstein, G., \& Shelley, C. (1981). Does the right hemisphere age more rapidly than the left? Journal of Clinical Neuropsychology, 3, 65-78.

Hale, S., Lima, S.D., \& Myerson, J. (1991). General cognitive slowing in the nonlexical domain: An experimental validation. Psychology and Aging, 6, $512-521$.

Heim, A. (1970). A4H test: Group test of intelligence. Windsor, Canada: NFER.

Humphreys, G.W., \& Riddoch, M.J. (1984). Routes to object constancy: Implications from neurological impariments of object constancy. Quarterly Journal of Experimental Psychology, 36A, 385-415.

Klisz, D. (1978). Neuropsychological evaluation in older persons. In M. Storandt, I.C. Siegler, \& M.D. Lezak (Eds.), The clinical psychology of aging (pp. 71-96). New York: Plenum Press.

Kosslyn, S.M. (1987). Seeing and imaging in the cerebral hemispheres: A computational approach. Psychological Review, 94, 148-175.

Kosslyn, S.M., \& Koenig, O. (1992). Wet mind: The new cognitive neuroscience. New York: Free Press.

Kosslyn, S.M., Maljkovic, V., Hamilton, S.E., Horwitz, G., \& Thompson, W.L. (1995). Two types of image generation: Evidence for left and right hemisphere processes. Neuropsychologia, 33, 1485-1510.

Lamb, L.C., \& Robertson, M.R. (1988). The processing of hierarchical stimuli: Effects of retinal loci, locational uncertainty, and stimulus identity. Perception and Psychophysics, 44, 172-181.

Lawrence, B.M., Myerson, J., \& Hale, S. (1997, March). Processing speed across the adult life span. Poster presented at the Annual Meeting of the Cognitive Neuroscience Society, Boston, MA.

Levine, S.C., \& Banich, M.T. (1982). Lateral asymmetries in the naming of words and corresponding line drawings. Brain and Language, 17, 34-45.

Levy, J. (1969). Possible basis for the evolution of lateral specialization of the human brain. Nature, 224, 614-615.

Lima, S.D., Hale, S., \& Myerson, J. (1991). How general is general slowing? Evidence from the lexical domain. Psychology and Aging, 6, 416-425.
Marsolek, C.J. (1995). Abstract visual-form representations in the left cerebral hemisphere. Journal of Experimental Psychology: Human Perception \& Performance, 21, 375-386.

Meudell, P.R., \& Greenhalgh, M. (1987). Age-related differences in left and right hand skill and in visuospatial performance: Their possible relationships to the hypothesis that the right hemisphere ages more rapidly than the left. Cortex, 23, 431-445.

Milner, B. (1975). Psychological aspects of focal epilepsy and its neurosurgical management. In D. Purpura, J. Penry, \& R. Walters (Eds.), Advances in neurology (Vol. 8, pp. 299-319). New York: Raven Press.

Myerson, J., Ferraro, F.R., Hale, S., \& Lima, S.D. (1992). General slowing in semantic priming and word recognition. Psychology and Aging, 7, 257270.

Navon, D. (1977). Forest before trees: The precedence of global features in visual perception. Cognitive Psychology, 9, 353-383.

Nebes, R.D. (1974). Hemispheric specialization in commissurotomized man. Psychological Bulletin, 81, 1-14.

Nebes, R.D., Madden, D.J., \& Berg, W.D. (1983). The effect of age on hemispheric asymmetry in visual and auditory identification. Experimental Aging Research, 9, 87-91.

Oldfield, R.C. (1971). The assessment and analysis of handedness: The Edinburgh Inventory. Neuropsychologia, 9, 97-114.

Polster, M.R., \& Rapcsak, S.Z. (1994). Hierarchical stimuli and hemispheric specialization: Two case studies. Cortex, 30, 487-497.

Riddoch, M.J., \& Humphreys, G.W. (1986). Neurological impariments of object constancy: The effects of orientation and size disparities. Cognitive Neuropsychology, 3, 207-224.

Robertson, L.C., Lamb, M.R., \& Zaidel, E. (1993). Interhemispheric relations in processing hierarchical patterns: Evidence from normal and commissurotomized subjects. Neuropsychology, 7, 325342.

Sergent, J., Ohta, S., \& MacDonald, B. (1992). Functional neuroanatomy of face and object processing. Brain, 115, 15-36.

Snodgrass, J.G., \& Corwin, J. (1988). Pragmatics of measuring recognition memory: Applications to dementia and amnesia. Journal of Experimental Psychology: General, 117, 34-50.

Stark, M.E., \& Coslett, H.B. (1993). The "Navon effect"'-Forest before trees?: It depends on how old you are and how many trees are in the forest. Journal of Clinical and Experimental Neuropsychology, 15, 46 (abstract).

Warrington, E.K. (1982). Neuropsychological studies of object recognition. Philosophical Transactions of the Royal Society of London B, 298, 15-33. 
Warrington, E.K., \& James, M. (1988). Visual apperceptive agnosia: A clinico-anatomical study of three cases. Cortex, 24, 13-32.

Warrington, E.K., \& Taylor, A.M. (1978). Two categorical stages of object recognition. Perception, 7 , 695-709.
Wechsler, D. (1958). The measurement and appraisal of adult intelligence. Baltimore: Williams and Wilkins. 